\title{
Ward Engagement Study: a teaching strategy to enhance pharmacy Students' integration into the ward environment
}

Author: Natalie Lewis, University Hospitals Birmingham NHS Foundation Trust

Background: The demand for hospitals to provide work based learning is increasing due to a widening pool of pharmacy undergraduates. This heavily supervised learning relies on trained pharmacists for delivery but often gives little opportunity for the student to integrate into the ward environment.

Method: This study sought to explore a new method for learning that could lift the burden on staff time whilst enhancing ward engagement. All $3^{\text {rd }}$ year pharmacy vacation students $(n=8)$ at one UK hospital were enrolled to complete one week of ward engagement activities on a gastroenterology ward under remote supervision (where the pharmacist supervisor was available on the ward and contactable at all times) allowing integration into the daily ward operations and interaction with patients and healthcare professionals. The students were supported by a workbook of activities which included: shadowing professionals and ward rounds, bed space comparisons, intervention reviews, ward communication tool comparison and patient interviews. The strategy was evaluated by comparison of students' attitudes pre and post participation via questionnaire and informal discussion. Impact on the ward was assessed by questionnaire that was completed by staff interacting with the students.

Results: The majority of workbook tasks were completed by the students (average $98 \%)$. All participants $(100 \%, n=8)$ were satisfied with the level of supervision and felt the experience met their expected learning objectives. Feedback forms were completed by approximately half $(50 \%, \mathrm{n}=15)$ of ward staff that interacted with the students. These showed no adverse effect on their duties $(76 \%, n=9)$, with approximately a quarter stating the presence of the students enhanced their normal duties $(24 \%, n=6)$.

Conclusion: This study demonstrates that a variety of learning objectives and increased ward engagement could be delivered through remote supervision of students in a healthcare setting. Student experiences in hospital can occur without the associated cost of intense supervision. 